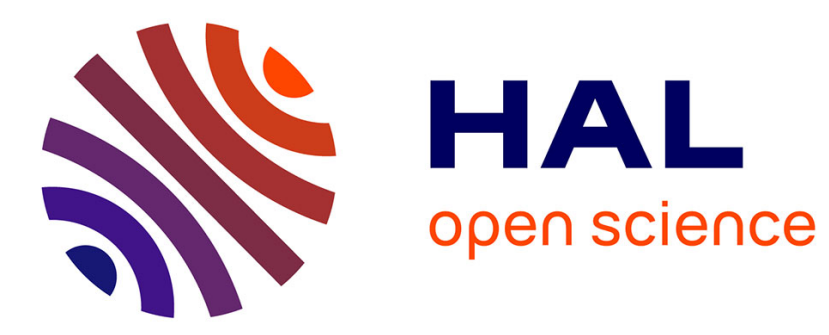

\title{
The combined Lagrangian advection method
}

David Dritschel, Jerome Fontane

\section{To cite this version:}

David Dritschel, Jerome Fontane. The combined Lagrangian advection method. Journal of Computational Physics, 2010, vol. 229, pp. 5408-5417. 10.1016/j.jcp.2010.03.048 . hal-00786319

\section{HAL Id: hal-00786319 https://hal.science/hal-00786319}

Submitted on 8 Feb 2013

HAL is a multi-disciplinary open access archive for the deposit and dissemination of scientific research documents, whether they are published or not. The documents may come from teaching and research institutions in France or abroad, or from public or private research centers.
L'archive ouverte pluridisciplinaire HAL, est destinée au dépôt et à la diffusion de documents scientifiques de niveau recherche, publiés ou non, émanant des établissements d'enseignement et de recherche français ou étrangers, des laboratoires publics ou privés. 


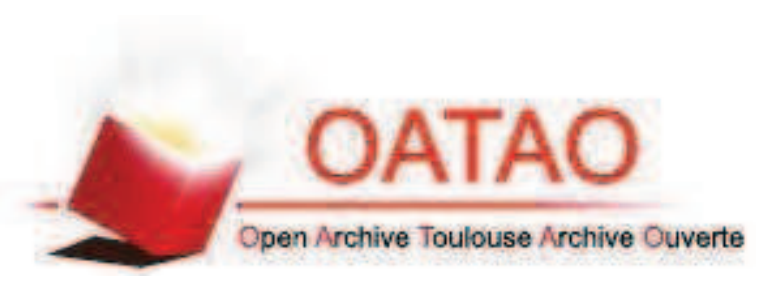

Open Archive Toulouse Archive Ouverte (OATAO)

OATAO is an open access repository that collects the work of Toulouse researchers and makes it freely available over the web where possible.

This is an author-deposited version published in: http://oatao.univ-toulouse.fr/ Eprints ID: 8396

To link to this article: DOI: $10.1016 / \mathrm{j} . j \mathrm{cp} .2010 .03 .048$

URL: http://dx.doi.org/10.1016/j.jcp.2010.03.048

To cite this version: Dritschel, David and Fontane, Jérôme The combined Lagrangian advection method. (2010) Journal of Computational Physics, vol. 229 ( $\left.\mathrm{n}^{\circ} 14\right)$. pp. 5408-5417. ISSN 0021-9991

Any correspondence concerning this service should be sent to the repository administrator: staff-oatao@inp-toulouse.fr 


\title{
The Combined Lagrangian Advection Method
}

\author{
David G. Dritschel and Jérôme Fontane \\ School of Mathematics and Statistics, University of St Andrews, St Andrews KY16 \\ 9SS, Scotland
}

\begin{abstract}
We present and test a new hybrid numerical method for simulating layerwise-twodimensional geophysical flows. The method radically extends the original ContourAdvective Semi-Lagrangian (CASL) algorithm (Dritschel \& Ambaum, 1997) by combining three computational elements for the advection of general tracers (e.g. potential vorticity, water vapor, etc.): (1) a pseudo-spectral method for large scales, (2) lagrangian contours for intermediate to small scales, and (3) lagrangian particles for the representation of general forcing and dissipation. The pseudo-spectral method is both efficient and highly accurate at large scales, while contour advection is efficient and accurate at small scales, allowing one to simulate extremely fine-scale structure well below the basic grid scale used to represent the velocity field. The particles allow one to efficiently incorporate general forcing and dissipation.
\end{abstract}

Key words:

pseudo-spectral, contour advection, vortex methods, two-dimensional turbulence

\section{Introduction}

Numerical simulations of atmospheric and oceanic fluid dynamics must deal with a vast range of active spatial and temporal scales of motion. Much of this range lies beyond the limit of numerical resolution, requiring small scales (and high frequencies) to be instead parametrised by 'eddy-diffusivity' or 'closure' schemes meant to approximate the collective effects of unresolved motions on resolved ones (cf. Nadiga, 2008 \& refs.). Here, we describe a new modelling advance which reduces the need for closure schemes by allowing one to efficiently extend the range of resolved scales, in particular for advected tracers. The new advance is the culmination of years of model development based on "Contour Advection" (CASL, Dritschel \& Ambaum, 1997), a hybrid Lagrangian-Eulerian method stemming originally from "Contour Surgery" (Dritschel, 1988) and "Contour Dynamics" (Zabusky, Hughes 
\& Roberts, 1979). The new method, called the "Combined Lagrangian Advection Method" (CLAM), utilises three computational elements - contours, particles, and grid points (or spectral coefficients) - combined in a way to optimise performance and accuracy.

While CLAM is built for accurate conservation in the absence of forcing and dissipation, it also allows one to efficiently handle general non-conservative processes such as thermal heating, Ekman friction, stochastic forcing, etc (Dritschel \& Ambaum, 2006; Mohebalhojeh \& Dritschel, 2009; Fontane \& Dritschel, 2009). Moreover, it may offer distinct advantages over commonlyused numerical methods in Geophysical Fluid Dynamics when multiple tracers (dynamical, chemical, biological) are considered (see below).

In the next section, we discuss the structure of CLAM. The method is illustrated and tested in an example of forced two-dimensional turbulence in $\S 3$. Finally, some conclusions and ideas for further model development are offered in $\S 4$.

\section{The method}

CLAM was developed originally to better model both unforced and forced 2D turbulence at ultra-high Reynolds numbers (Dritschel et al, 2008, 2009; Dritschel \& Scott, 2009; Fontane \& Dritschel, 2009). It is an extension of the recent HyperCASL algorithm (Fontane \& Dritschel, 2009), which introduced the idea of using point vortices or particles to represent a residual tracer field $q_{d}$ (e.g. vorticity in 2D turbulence or potential vorticity in rotating stratified flows). The residual field $q_{d}$ is used as a temporary reservoir for any explicit forcing and dissipation: approximately every 4 eddy-turnaround times $T_{\text {eddy }}$ (determined from the maximum vorticity integrated over time), $q_{d}$ is transferred to a set of contours representing the primary tracer field $q_{a}$ through an efficient contouring procedure (Dritschel \& Ambaum, 2006). At any instant of time $t$, the full tracer field $q$ is the sum

$$
q=q_{a}+q_{d}
$$

in which $q_{a}$ evolves conservatively by contour advection, i.e.

$$
\frac{\mathrm{d} \boldsymbol{X}_{a}}{\mathrm{~d} t}=\boldsymbol{u}\left(\boldsymbol{X}_{a}, t\right)
$$

(equivalent to $\mathrm{D} q_{a} / \mathrm{D} t=\partial q_{a} / \partial t+\boldsymbol{u} \cdot \boldsymbol{\nabla} q_{a}=0$ ), where $\boldsymbol{X}_{a}$ is a point on a contour and $\boldsymbol{u}(\boldsymbol{x}, t)$ is the velocity field, while $q_{d}$ evolves by advecting discrete particles $\boldsymbol{X}_{d}$

$$
\frac{\mathrm{d} \boldsymbol{X}_{d}}{\mathrm{~d} t}=\boldsymbol{u}\left(\boldsymbol{X}_{d}, t\right)
$$



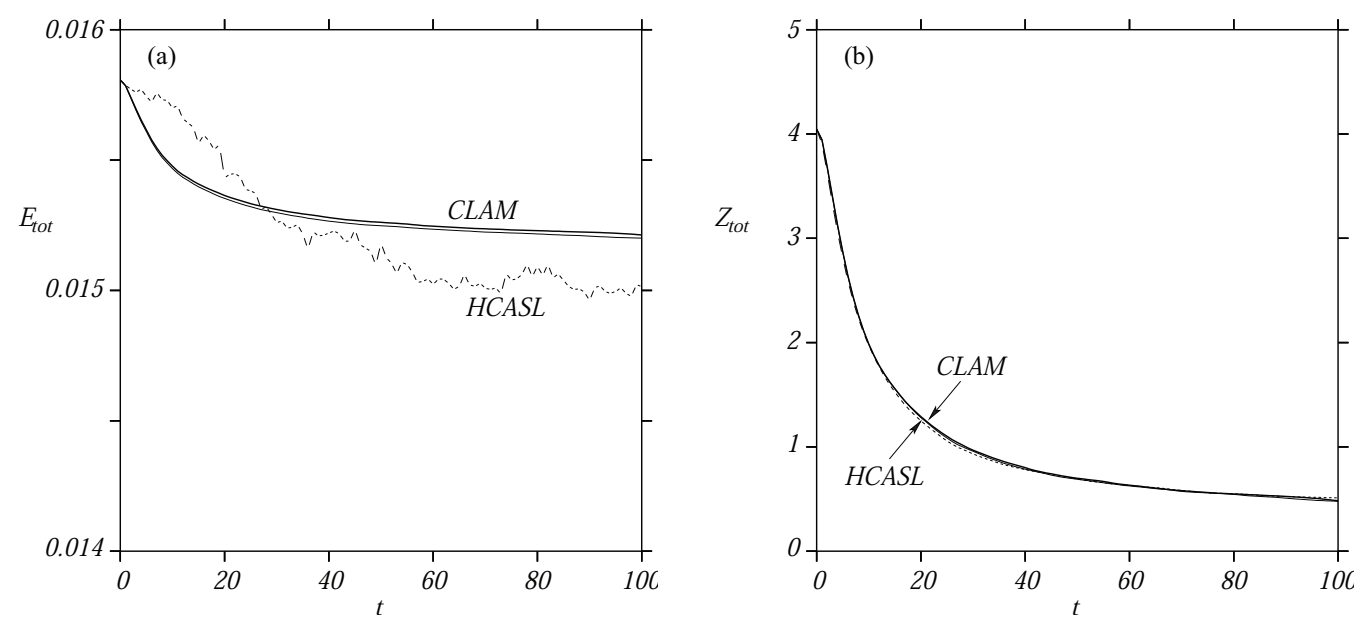

Fig. 1. Comparison of the evolution of (a) energy $E_{\text {tot }}(t)$ (normalised by $\left.E_{\text {tot }}(0)=0.0148588\right)$ and (b) enstrophy $Z_{\text {tot }}(t)$ (normalised by $\left.Z_{\text {tot }}(0)=4.046645\right)$ between HyperCASL (dashed) and CLAM (solid) in the case of freely decaying two-dimensional turbulence examined in Fontane \& Dritschel (2009). The two curves shown for CLAM correspond to two different ways of representing $q_{d}$, by particles (bold) or by a spectral method (thin). See text below for details.

and adjusting the intensities $\Gamma_{d}$ of individual particles to match the imposed forcing and dissipation (for details, see Fontane \& Dritschel, 2009). Weak numerical dissipation occurs during the regularization of contours by "surgery" (Dritschel, 1988), and when resetting the particles on a regular array. Both are done approximately every $0.2 T_{\text {eddy }}$. Notably, this dissipation is comparable to that needed in a conventional spectral method using a grid 10-20 times finer than used in HyperCASL and CLAM to represent the velocity field $\boldsymbol{u}$ (Dritschel \& Scott, 2009).

An unwanted feature of HyperCASL is the introduction of a small level of stochastic noise in $q_{a}$ by the contour-to-grid conversion procedure and, to a much lesser extent, by contour surgery (Fontane \& Dritschel, 2009). Unfortunately, this noise is statistically uniform across Fourier modes and it generates a growing $k^{1}$ tracer variance spectrum at small $k$. In simulations of $2 \mathrm{D}$ turbulence (Fontane \& Dritschel, 2009), this gives rise to a growing $k^{-1}$ energy spectrum at small $k$ (the actual energy spectrum normally decays rapidly as $k \rightarrow 0$, see e.g. Dritschel et al, 2009). As a consequence, this noise primarily affects the energy while enstrophy (vorticity variance) is more robust, see figures 1 and 2. And in long-time simulations, it can eventually lead to significant erroneous energy loss.

CLAM removes this unwanted feature (see figure 1(a)) by using a pseudospectral (PS) method to model large scales, specifically wavenumbers $k \leq k_{c}$, where $k_{c}$ is the 'filter cutoff wavenumber'. The PS method is well-designed for this purpose and, moreover, is both accurate and efficient. In CLAM, the tracer field computed this way, denoted $q_{s}$, is blended with the primary tracer 

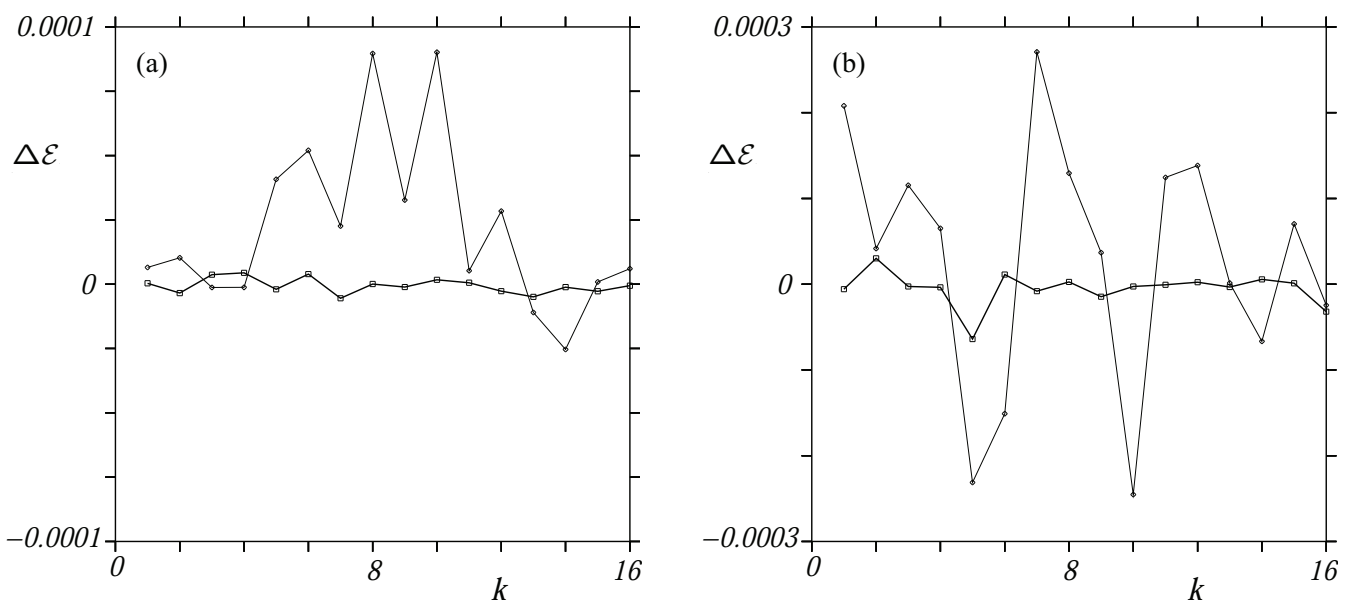

Fig. 2. Difference energy spectra $\Delta \mathcal{E}(k, t)$ as a function of wavenumber $k$, at low $k$, between HyperCASL and CLAM with particles (thin solid line with diamonds) and between CLAM with spectral $q_{d}$ and CLAM with particles (bold solid line with squares), at (a) $t=2$ and (b) $t=5$. Note, $\mathcal{E}(k, t)$ is sum of the energy $|\hat{\boldsymbol{u}}|^{2} / 2$ in spectral components with wavevectors $\boldsymbol{k}$ satisfying $|\boldsymbol{k}|=k=1,2, \ldots$ (nearest integer). The results were obtained from the turbulence simulations examined in figure 1.

field $q_{a}$ (represented by contours). The full tracer field is obtained now from

$$
\hat{q}=F \hat{q}_{s}+(1-F) \hat{q}_{a}+\hat{q}_{d}
$$

where a hat denotes a spectral transform, and $F(k)$ is a low-pass filter (see below). The only difference between HyperCASL and CLAM is the replacement of $q_{a}$ in (1) by a weighted sum of $q_{s}$ and $q_{a}$. The form of the low-pass filter $F(k)$ was fixed after extensive numerical tests (see below), and it takes the form

$$
F(k)=\frac{1}{1+\left(k / k_{c}\right)^{4}} .
$$

This is known as the second-order Butterworth filter (Butterworth, 1930), and it has a maximal flatness property for $k \leq k_{c}$. Additionally, there is a smooth transition between $\hat{q}_{s}$ and $\hat{q}_{a}$ around the filter cutoff wavenumber $k_{c}$.

The introduction of $q_{s}$ requires another dynamical evolution equation. Here, we evolve $q_{s}$ conservatively, i.e.

$$
\frac{\mathrm{D} q_{s}}{\mathrm{D} t}=0
$$

- with no dissipation whatsoever. This is of course unsustainable in general, as $\hat{q}_{s}$ will cascade to high $k$ by advection, and the solution will become unphysical if these high $k$ components of $\hat{q}_{s}$ are not removed, e.g. by numerical diffusion. Instead, in CLAM, $q_{s}$ and $q_{d}$ are reinitialised at the beginning of every time-step. The field $q_{s}$ is set to the entire $q$ field obtained at the end of the previous time-step. Meanwhile, the field $q_{d}$ is chosen to ensure that $q$ 
is unchanged when $q_{s}, q_{a}$ and $q_{d}$ are next recombined. Mathematically, this means taking

$$
\hat{q}_{s}=\hat{q} \quad \text { and } \quad \hat{q}_{d}=(1-F)\left(\hat{q}-\hat{q}_{a}\right)
$$

where $\hat{q}$ is the (spectral) field at the end of the previous time-step. When they are next recombined, we find

$$
F \hat{q}_{s}+(1-F) \hat{q}_{a}+\hat{q}_{d}=F \hat{q}+(1-F) \hat{q}_{a}+(1-F)\left(\hat{q}-\hat{q}_{a}\right)=\hat{q}
$$

as required. A fourth-order Runge-Kutta procedure is convenient for the subsequent integration over a single time-step.

This reinitialisation procedure avoids the erroneous random variations of $\hat{q}_{a}$ across all $k$ and moreover allows an accurate estimate of the advection term $\boldsymbol{u} \cdot \boldsymbol{\nabla} q_{s}$ needed for evolving $\hat{q}_{s}$. Furthermore, any forcing or dissipation in $\hat{q}_{d}$ in wavenumbers $k \lesssim k_{c}$ is transferred every time-step to $\hat{q}_{s}$, thereby minimizing numerical diffusion in $\hat{q}_{d}$. This is especially important for forced flows, as we shall see in $§ 3$. Regarding efficiency, in forced flows CLAM is only a few percent slower than HyperCASL and significantly more accurate (a comparison is provided below). The extra cost associated with the spectral method, at the resolution required for its use in CLAM, is minimal due to the spectral method's particularly high efficiency compared to the contour and particle methods.

The reinitialisation of $q_{d}$ does however require a transfer of the gridded $q_{d}$ to the particle intensities $\Gamma_{d}$. This is not an efficient procedure but is required every time-step in forced flows. In unforced flows, such as considered in Fontane \& Dritschel (2009) for HyperCASL, this transfer is required only when the particle array is reset - in practice only after many time-steps. As a result, HyperCASL is relatively efficient, approximately $60 \%$ faster than CLAM in the simulation of unforced 2D turbulence illustrated in figure 1 (discussed in Fontane \& Dritschel, 2009). We can gain back this loss of efficiency, and more, if we replace the particle method for $q_{d}$ by a spectral method (as in DCASL, see Dritschel \& Ambaum, 2006, and Mohebalhojeh \& Dritschel, 2009) when simulating unforced flows, or flows forced or damped predominantly at large scales (e.g. thermal forcing in geophysical flows). But the use of a spectral method for $q_{d}$ requires some sort of numerical diffusion for stability. The simplest approach is hyperdiffusion, i.e.

$$
\frac{\mathrm{D} q_{d}}{\mathrm{D} t}=\nu_{d}(-1)^{p+1} \nabla^{2 p} q_{d}
$$

and after extensive tests varying both the power $p$ and the coefficient $\nu_{d}$, we have adopted the choice $p=2$ (bi-harmonic hyperviscosity) together with $\nu_{d}=5 \zeta_{\mathrm{rms}}(t) / k_{m}^{4}$, in which $k_{m}$ is the maximum wavenumber associated with the inversion grid and $\zeta_{\mathrm{rms}}$ is the r.m.s. (vertical) vorticity. Besides maintaining numerical stability and greatly reducing Gibbs fringes near high gradients in 
Table 1

\begin{tabular}{|c|c|}
\hline Numerical Algorithm & Cost (CPU seconds) \\
\hline HyperCASL & 2669 \\
CLAM with PS $q_{d}$ & 2459 \\
CLAM with PIC $q_{d}$ & 4285 \\
\hline
\end{tabular}

Comparison of algorithm efficiency (using a $2.8 \mathrm{GHz}$ Intel processor) in a simulation of freely-decaying 2D turbulence, cf. figure 1 and Fontane \& Dritschel (2009). Here, HyperCASL is compared with two versions of CLAM, the first using a pseudospectral (PS) method for $q_{d}$ and the second using a particle-in-cell (PIC) method. Here, CLAM with PS $q_{d}$ is $8 \%$ more efficient than HyperCASL, while CLAM with PIC $q_{d}$ is $60 \%$ less efficient.

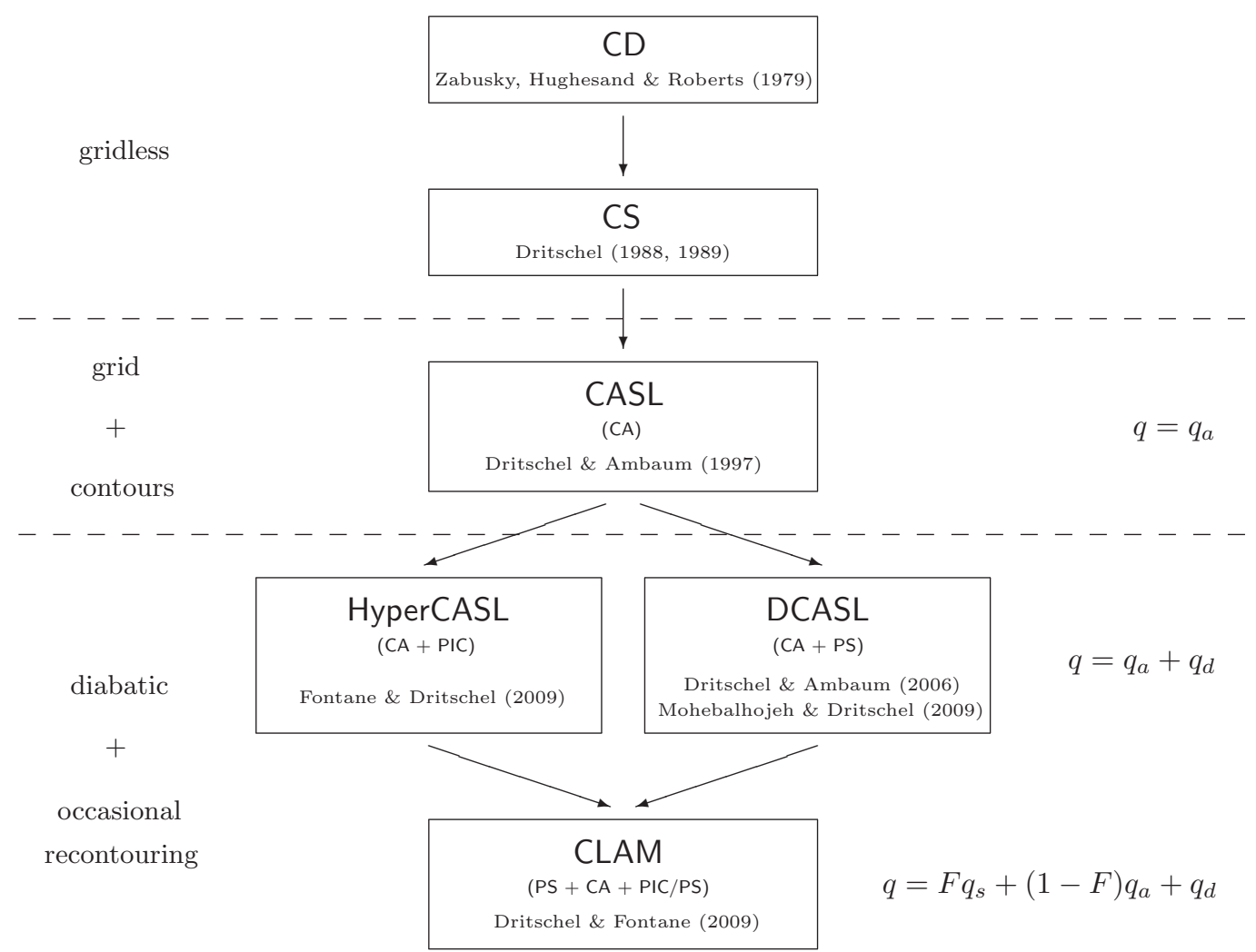

Fig. 3. A genealogical tree for numerical methods based wholly or in part on contour advection. Here $\mathrm{CD}=$ contour dynamics, $\mathrm{CS}=$ contour surgery, DCASL = diabatic CASL, PS $=$ pseudo-spectral and PIC $=$ particle-in-cell

$q_{d}$, this choice of hyperdiffusion gives the closest comparison to the results we find when using particles for $q_{d}$. Using a spectral method for $q_{d}$ rather than particles is guaranteed to be more efficient. In the unforced simulation illustrated in figure 1, this spectral version of CLAM results in a nearly $10 \%$ gain in efficiency over HyperCASL - see Table 1 - and achieves significantly greater accuracy. Although CLAM with PS $q_{d}$ is slightly less accurate than CLAM with PIC $q_{d}$, it is nearly twice as efficient. 

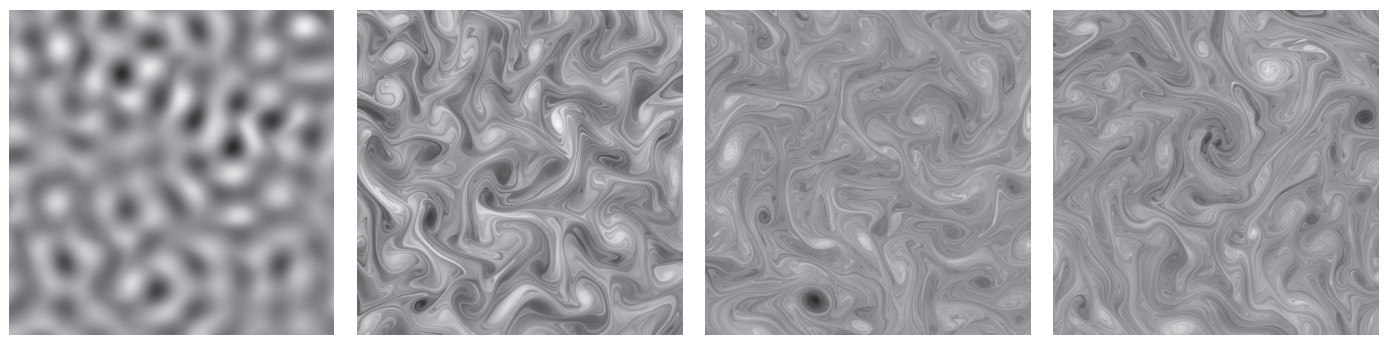

Fig. 4. Evolution of the vorticity field in a CLAM simulation of forced 2D turbulence using a basic $256 \times 256$ grid (contours are retained to scales 16 times smaller). Times $t=2,5,10$ and 20 are shown from left to right. Only $1 / 16$ th of the domain is shown. A linear grey scale is used between $-|q|_{\max }<q<|q|_{\text {max }}$ (black being most negative).

A small modification of the re-contouring procedure in HyperCASL (Fontane \& Dritschel, 2009) was made in CLAM, following extensive tests, to improve the accuracy of this procedure. In recontouring, the difference field $q^{\prime}=q-q_{a}$ at the end of a simulation period is extrapolated from the basic 'inversion' grid to an ultra-fine contouring grid (16 times finer in each direction) and added to the contour-associated field $q_{a}$ on this grid (whose smallest scale is the scale of surgery). This combined field is then recontoured, and the error in contouring with a finite contour interval $\Delta q$ present on the inversion grid is given to $q_{d}$ to start the next simulation period. There is then no change to the total $q$ on the inversion grid following recontouring. In HyperCASL, we used bi-linear interpolation to extrapolate $q^{\prime}$ to the contouring grid, whereas in CLAM (and now in HyperCASL too) we use spectral interpolation (zero padding) for greater accuracy. In this way we retain all information in $q^{\prime}$. Spectral interpolation results in no significant loss in efficiency.

A brief history of the key developments leading to CLAM is provided in figure 3 .

\section{A test case}

We next examine a numerical simulation of forced $2 \mathrm{D}$ turbulence, and use this to justify our choice of the filter $F(k)$ and the filter cutoff wavenumber $k_{c}$. Note, here $q$ is the 2D (barotropic) vorticity. We have carried out simulations at three different resolutions and for widely varying forcing wavenumbers $k_{f}$. Following many previous studies, we have used narrow-band forcing in which a randomphased top-hat enstrophy spectrum, constant over $k_{f}-\Delta k \leq k \leq k_{f}+\Delta k$, is added every time-step to ensure a constant rate of enstrophy growth, here 7.8957, in the absence of dissipation. This forcing results in a nearly linear growth of energy, which is only weakly dissipated over the times considered. Note, the flow starts from a state of rest. 


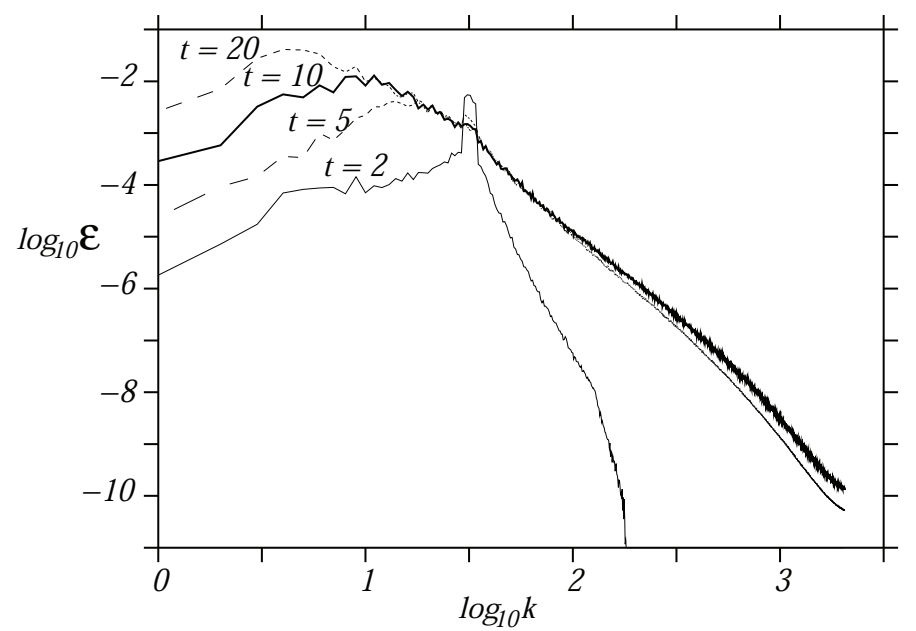

Fig. 5. Energy spectra $\mathcal{E}(k, t)$ at the times corresponding to the images in figure 4 . Note that the spectral tails extend well beyond the maximum wavenumber $k_{m}=128$ associated with the inversion grid. These tails have slopes between $k^{-4}$ and $k^{-3}$, and do not change significantly after $t=10$. Instead, the spectra build at low wavenumbers (large scales), consistent with a linear growth in total energy (see text).

We focus on one simulation with a basic 'inversion grid' of $256 \times 256$ (with maximum wavenumber $\left.k_{m}=128\right)$, forced at $k=k_{f}=32$ (with $\Delta k=2$ ), and using all of the standard parameter settings recommended in Fontane \& Dritschel (2009). In particular, we use a contour interval $\Delta q=\pi / 5 \approx 0.6283$ for representing $q_{a}$ (using an estimated unit eddy turnaround time). The evolution is simulated over a moderate time period, $0 \leq t \leq 20$, corresponding to nearly $80 T_{\text {eddy }}$ based on the time integrated maximum vorticity, or $10.5 T_{\text {eddy }}$ based on the time integrated r.m.s. vorticity. A few stages in the evolution (at $t=2,5,10$ and 20) are shown in figure 4 (note, only 1/16th of the domain is shown). In the earliest stage shown (upper left), the direct effect of the forcing is most evident; the flow has not yet had time to evolve significantly. By the next stage, one can already see the formation of vortices surrounded by cascading filamentary debris. The later stages are closely similar, albeit with more fine-scale structure and marginally more organised large-scale vortices (the energy grows by cascading slowly to large scales from the forcing wavenumber).

In spectral space (see figure 5 ), energy $\mathcal{E}(k, t)$ (shell averaged over wavevectors $\boldsymbol{k}$ of magnitude $k=|\boldsymbol{k}|$ ) spreads from its initial forcing region near $k=k_{f}=32$ and rapidly becomes nearly time-independent except at small $k$. There, energy grows as it cascades to large scales, resulting in a growth of the total energy $E_{\text {tot }}(t)=\int \mathcal{E}(k, t) \mathrm{d} k$. This energy growth is approximately linear in time, as expected statistically from narrow-band random forcing at large $k_{f}$. Figure 6 compares the observed growth for various simulations with the theoretical linear growth (dotted line). Two CLAM simulations are illustrated, one using 


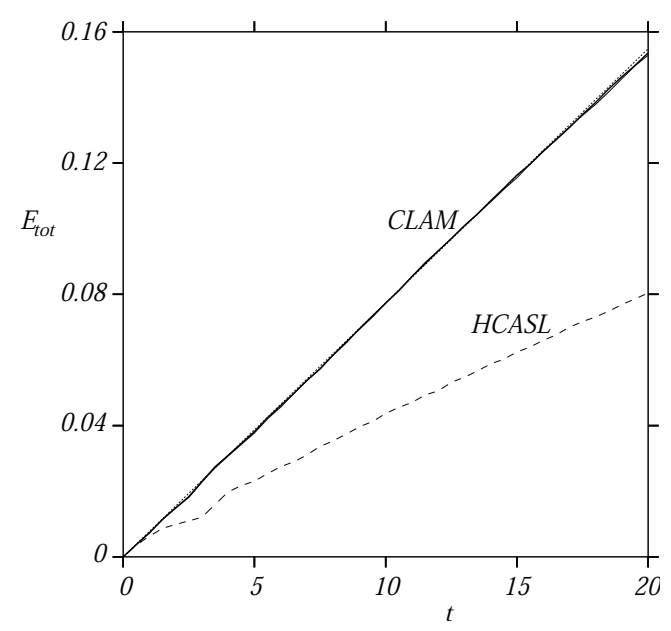

Fig. 6. Total energy $E_{\text {tot }}(t)$ versus time in several simulations of forced 2D turbulence, with forcing centred on wavenumber $k=k_{f}=32$. The bold, thin and dashed curves correspond to CLAM using particles for $q_{d}$, CLAM using a spectral method for $q_{d}$, and HyperCASL, respectively, while the dotted line gives the theoretical growth. This simulations use all the standard numerical parameters set out in Fontane \& Dritschel (2009). In addition, the CLAM simulations here use the second-order Butterworth filter (5) with cutoff wavenumber $k_{c}=k_{m} / 3=42$.
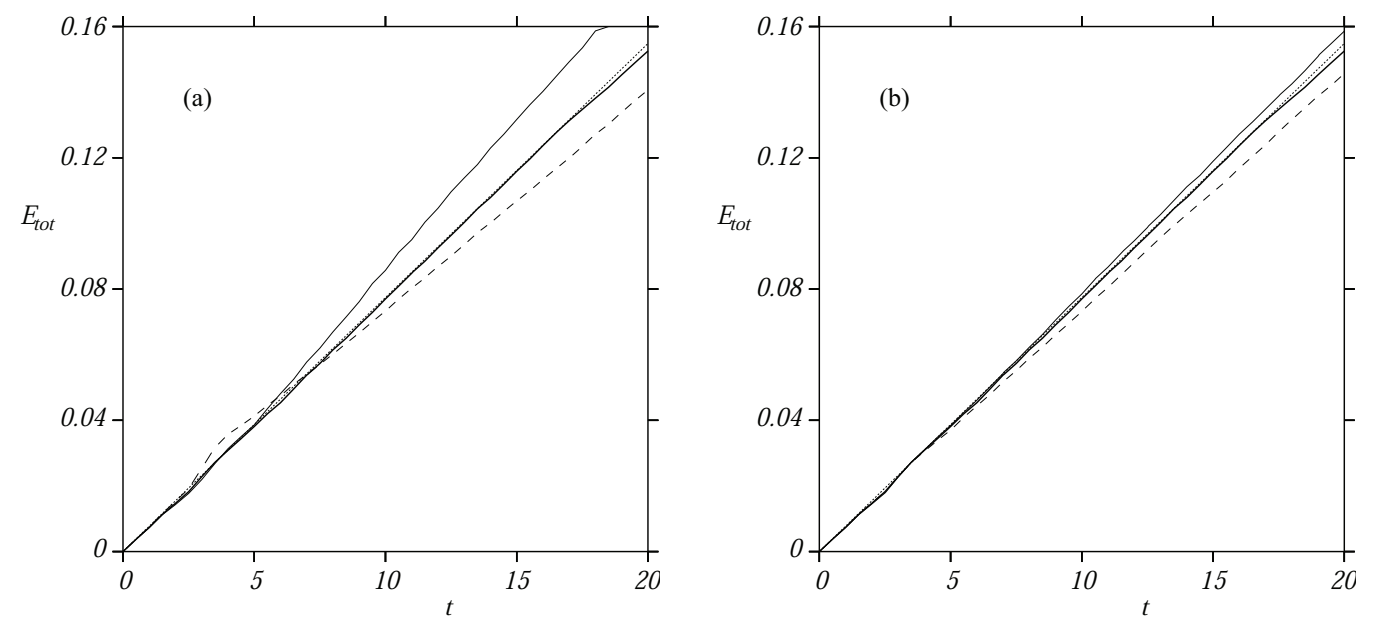

Fig. 7. (a) Total energy $E_{\text {tot }}(t)$ versus time for three CLAM simulations using the second-order Butterworth filter (5) with cutoff wavenumbers $k_{c}=32$ (thin line), 42 (bold) and 64 (dashed). The theoretical prediction is given by the straight dotted line. (b) As in (a) except now for $k_{c}=42$ and for the following filters $F(k)$ : first-order Butterworth $\left[1+\left(k / k_{c}\right)^{2}\right]^{-1}$ (thin line), second-order Butterworth $\left[1+\left(k / k_{c}\right)^{4}\right]^{-1}$ (bold), and third-order Butterworth $\left[1+\left(k / k_{c}\right)^{6}\right]^{-1}$ (dashed).

particles for $q_{d}$ (bold line) and the other using a spectral method for $q_{d}$ (thin line). The HyperCASL simulation (lower dashed line) shows poor conservation properties. The CLAM simulations, by contrast, closely match the theoretical growth apart from a slight deficit at early times, during the first period of integration from rest without contours $(0 \leq t \leq 2.846)$. 
Figure 7 compares the energy growth in CLAM simulations using (a) different filter cutoff wavenumbers $k_{c}$ for the same second-order Butterworth filter (5), and (b) different orders of the Butterworth filter for the same cutoff wavenumber, $k_{c}=42\left(\approx k_{m} / 3\right)$. We see in (a) that $k_{c}=42$ gives the best fit to the theoretical prediction, while in (b) that the second-order Butterworth filter gives the best fit. Poorer results (not shown) were found for the Gaussian filter $F(k)=e^{-\left(k / k_{c}\right)^{2}}$ and the sharp filter $F(k)=1$ for $k \leq k_{c}$ and $F(k)=0$ otherwise. The dependence on $k_{c}$ can be explained by noting that, for small $k_{c}$, most of the dynamics is controlled by the contours in $q_{a}$, and we know from Fontane \& Dritschel (2009) that contour advection alone can lead to an erroneous growth of energy at low wavenumbers, resulting in an enhanced growth of $E_{\text {tot }}$. Conversely, for large $k_{c}$, most of the dynamics is controlled by the spectral evolution which is more diffusive than contour advection, resulting in a retarded growth of $E_{\text {tot }}$. From these results, and many other simulations for different ratios of $k_{f} / k_{m}$, we have found that the second-order Butterworth filter with a filter cutoff wavenumber $k_{c}=k_{m} / 3$ is most accurate.

There is a limit, however, to how large one can make the ratio $k_{f} / k_{m}$. Above, we examined $k_{f} / k_{m}=1 / 4$, but when using a larger value like $k_{f} / k_{m}=1 / 2$ we observed an early retarded growth of $E_{\text {tot }}$ followed by a later growth having a slope slightly lower than expected (not shown). Every effort was made to correct this growth, from modifying the numerical parameter settings in CLAM, to using a smaller contour interval $\Delta q$, to changing the bi-linear interpolation of the velocity field on contour points and particles to the third-order $M_{4}^{\prime}$ scheme of Cottet, Ould Sahili \& El Hamraoui (1999), or the incompressible quadratic-spline method of Handscomb (1984), and even to replacing CLAM with a full pseudo-spectral simulation (on a four times finer inversion grid) for the early simulation period. None of these improved the growth of $E_{\text {tot }}$, and all were more costly.

This limitation on the size of $k_{f} / k_{m}$ is, however, not surprising. When $k_{f}$ is only half $k_{m}$, there is little room for resolving the nonlinear spread of the forcing through $q_{d}$, and this results in numerical dissipation. Relative to the maximum effective wavenumber associated with the ultra-fine grid, here $16 k_{m}$, our tests show that $k_{f}$ should be at least 64 times smaller. Independently, using a pure pseudo-spectral method, Scott (2007) has found that the maximum wavenumber must be at least 64 times the forcing wavenumber to properly model the inverse cascade in two-dimensional turbulence. It is therefore reasonable to limit $k_{f} / k_{m}$ to $1 / 4$ in CLAM when narrow-band spectral forcing is used. Other common types of forcing, such as thermal forcing in geophysical flows, tend to be broad-band with significant input at large scales. In general, these types of forcing can be more easily represented in numerical models, and CLAM is no exception. Indeed, CLAM like its predecessor HyperCASL offers new possibilities for forcing, e.g. by introducing new particles carrying circulation and other attributes (Fontane \& Dritschel, 2009). Such forcing may 
Table 2

\begin{tabular}{|c|c|}
\hline Numerical Algorithm & Cost (CPU seconds) \\
\hline HyperCASL & 7698 \\
CLAM with PS $q_{d}$ & 9856 \\
CLAM with PIC $q_{d}$ & 10864 \\
\hline
\end{tabular}

Comparison of algorithm efficiency in a simulation of forced 2D turbulence, cf. figure 4. In this case, both versions of CLAM are less efficient ( $28 \%$ and $41 \%$ respectively) than HyperCASL, but HyperCASL is unable to capture the correct linear energy growth.

be particularly effective in representing subgrid-scale convection in a model of the atmosphere.

As regards efficiency, CLAM is less efficient than its predecessor HyperCASL as indicated in Table 2. However, the extra work in CLAM is necessary, in the case of forced 2D turbulence analysed above, to accurately capture the correct growth in energy (see figure 6). The retarded energy growth seen in HyperCASL is a consequence of retaining the forcing wholly in particles, which diffuse vorticity every time they are reset on a regular array. By contrast, CLAM transfers most of the forcing, every time-step, to a spectral method (that used for $q_{s}$ ), thereby preserving nearly all of the added vorticity. This, we emphasize, is an especially important characteristic of CLAM. Hence, CLAM not only represents the large-scale dynamics optimally (i.e. with a spectral method), but also represents complex forcing accurately.

\section{Conclusions}

We have described and examined a new hybrid computational method, CLAM, combining three numerical elements: pseudo-spectral, contour advection and particle-in-cell. By using each element selectively across spatial scales, CLAM achieves a substantially greater efficiency and accuracy than is possible when using any one element on its own. This has been explicitly demonstrated for HyperCASL in Fontane \& Dritschel (2009), and CLAM is by design more accurate for little extra cost. The use of a pseudo-spectral (PS) method allows one to accurately represent the effects of forcing, and moreover avoids the slow, erroneous growth of large-scale modes caused by errors in the contour-to-grid conversion procedure and in contour surgery. The PS method is relatively efficient compared to contour advection and particle-in-cell, and therefore numerical efficiency is not significantly degraded. While the PS method is stable only for a time-step satisfying the CFL constraint $\Delta t<\Delta x /|\boldsymbol{u}|_{\max }$, in realistically complex geophysical flows the time-step is nearly always limited by the maximum vertical vorticity: $\Delta t<\pi / 10|\zeta|_{\max }$ (Fontane \& Dritschel, 2009). 
The CFL constraint applies only to flows dominated by large-scale motions.

CLAM is not limited to the study of two-dimensional turbulence. Like HyperCASL, it is presently capable of studying single and multi-layer 'quasigeostrophic' (rapidly-rotating, stably-stratified) geophysical flows subject to general forcing and dissipation mechanisms (cf. Fontane \& Dritschel, 2009, and Dritschel \& Fontane, 2010). CLAM has also been adapted to a periodic channel geometry, and work is in progress on a spherical extension. In different contexts, CLAM has been implemented to study two-dimensional rotating shallow-water flows, density-stratified flows in a vertical cross-section using density as the tracer $q$ (King, Carr \& Dritschel, 2010), and idealised magnetohydrodynamics where the (potential) vorticity $q$ is subject to the Lorenz force (Tobias, Diamond \& Dritschel, 2010).

Further extensions e.g. to three-dimensional rotating stratified flows are feasible, requiring straightforward adaptions of existing CASL codes (Dritschel \& Viúdez, 2003). CLAM also presents a promising opportunity for accurately modelling more realistic atmospheric and oceanic flows. CLAM can carry more than one tracer $q$. Additional chemical and biological tracers (those that are advection dominated) may be represented by additional sets of contours (with natural parallelisation possibilities). But, only one set of particles is required for all tracers. Each particle would contain a list of attributes, related to the residue $q_{d}$ belonging to each tracer. Any forcing or dissipation (including chemical reactions, changes of state, etc) would only modify the attributes of a particle, not its position, which is determined by simple advection, i.e. by (3). This appears to be a particularly efficient procedure for representing a collection of interacting tracers.

\section{Acknowledgments}

The authors wish to thank C. Macaskill for his comments and suggestions during the preparation of the manuscript. Jérôme Fontane is supported by the European Community under the CONVECT project (grant number PIEFGA-2008-221003).

\section{References}

Butterworth, S. 1930 On the theory of filter amplifiers. Experimental Wireless and the Wireless Engineer 7, 536-541.

Cottet, G. H., Ould Sahili, M.L. \& El Hamraoui, M. 1999 Multipurpose regridding in vortex methods. ESAIM Proc. Third international workshop on vortex flows and related numerical methods. 7, 94-103. 
Dritschel, D. G. 1988 Contour surgery: a topological reconnection scheme for extended integrations using contour dynamics. J. Comput. Phys. 77, 240-266.

Dritschel, D. G. \& Ambaum, M. H. P. 1997 A contour-advective semiLagrangian numerical algorithm for simulating fine-scale conservative dynamical fields. Quart. J. Roy. Meteorol. Soc. 123, 1097-1130.

Dritschel, D. G. \& Viúdez, Á. 2003 A balanced approach to modelling rotating stably-stratified geophysical flows. J. Fluid Mech. 488, 123-150.

Dritschel, D. G. \& Ambaum, M. H. P. 2006 The diabatic contour advective semi-Lagrangian algorithm. Mon. Wea. Rev. 134, 2503-2514.

Dritschel, D. G., Tran, C. V. \& Scott, R. K. 2007 Revisiting Batchelor's theory of two-dimensional turbulence. J. Fluid Mech. 591, 379-391.

Dritschel, D. G., Scott, R. K., Macaskill, C., Gottwald, G. A. \& Tran, C. V. 2008 Unifying scaling theory for vortex dynamics in twodimensional turbulence. Phys. Rev. Lett. 101, 094501.

Dritschel, D. G., Scott, R. K., Macaskill, C., Gottwald, G. A. \& Tran, C. V. 2009 Late time evolution of unforced inviscid two-dimensional turbulence. J. Fluid Mech. 640, 217-235.

Dritschel, D. G. \& ScotT, R. K. 2009 On the simulation of nearly inviscid two-dimensional turbulence. J. Comput. Phys. 228, 2707-2711.

Dritschel, D. G. \& Fontane, J. 2010 The HyperCASL Algorithm, In: Turbulence in the Atmosphere and Oceans, (Ed.: D.G. Dritschel), Springer (2010).

Fontane, J. \& Dritschel, D. G. 2009 The HyperCASL Algorithm: a new approach to the numerical simulation of geophysical flows. J. Comput. Phys. 228, 6411-6425.

Handscomb, D. C. 1984 Spline representation of incompressible flow. IMA J. Num. Ana. 4, 491-502.

King, S., Carr, M. \& Dritschel, D. G. 2010 On the steady state form of large amplitude internal solitary waves. J. Fluid Mech. (under review).

Mohebalhojeh, A. R. \& Dritschel, D. G. 2009 The diabatic contour advective semi-Lagrangian algorithms for the spherical shallow water equations. Mon. Wea. Rev. 137, 2979-2994.

NADigA, B. T. 2008 Orientation of eddy fluxes in geostrophic turbulence. Phil. Trans. R. Soc. A 366, 2491-2510.

Scott, R. K. 2007 Non-robustness of the two-dimensional turbulent inverse cascade. Phys. Rev. E 75, 046301.

Tobias, S. M., Diamond, P. H. \& Dritschel, D. G. 2010 The formation of MHD jets on a magnetised $\beta$-plane at finite deformation radius. Geophys. Astrophys. Fluid Dyn. (under review).

Zabusky, N. J., Hughes, M. \& Roberts, K. V. 1979 Contour dynamics for the Euler equations in two dimensions. J. Comput. Phys. 30, 96-106. 